\title{
On estimates of solutions of the periodic boundary value problem for first-order functional differential equations
}

Eugene Bravyi ${ }^{*}$

Dedicated to Professor Ivan Kiguradze

*Correspondence: bravyi@perm.ru

Scientific center 'Functional

Differential Equations', Perm

National Research Polytechnical

University, Komsomol'sky pr. 29,

Perm, 614990, Russia

\begin{abstract}
Inequalities for periodic solutions of first-order functional differential equations are obtained. These inequalities are best possible in a certain sense.
\end{abstract}

MSC: Primary 34K06; 34K10; 34K13

Keywords: functional differential equations; periodic solutions; periodic boundary value problem; estimates of solutions

\section{Introduction}

Periodic solutions of functional differential equations are important in different applications (see, for example, [1-4] and the references therein, and also works on the general theory of boundary value problems for functional differential equations [5-11]). Conditions for the solvability of first-order periodic problems are found in [12-23]. In [15, 16] the linear case is considered, and unimprovable sufficient conditions for the solvability of the periodic problem

$$
\begin{aligned}
& \dot{x}(t)=\left(T^{+} x\right)(t)-\left(T^{-} x\right)(t)+f(t), \quad t \in[a, b], \\
& x(a)=x(b),
\end{aligned}
$$

are found in terms of the norms $\mathcal{T}^{+}, \mathcal{T}^{-}$of linear positive functional operators $T^{+}, T^{-}$: $\mathrm{C} \rightarrow \mathrm{L}:$

$$
\frac{\mathcal{T}^{-}}{1-\mathcal{T}^{-}}<\mathcal{T}^{+}<2\left(1+\sqrt{1-\mathcal{T}^{-}}\right)
$$

or

$$
\frac{\mathcal{T}^{+}}{1-\mathcal{T}^{+}}<\mathcal{T}^{-}<2\left(1+\sqrt{1-\mathcal{T}^{+}}\right) .
$$

If both of these conditions are not satisfied for some norms $\mathcal{T}^{+}, \mathcal{T}^{-}$, there exist linear positive operators $T^{+}, T^{-}$with these norms such that problem (1)-(2) has no solution. As to our knowledge, similar unimprovable estimates for solutions of (1)-(2) in terms of norms

(O2014 Bravyi; licensee Springer. This is an Open Access article distributed under the terms of the Creative Commons Attribution License (http://creativecommons.org/licenses/by/2.0), which permits unrestricted use, distribution, and reproduction in any medium, provided the original work is properly cited. 
$\mathcal{T}^{+}, \mathcal{T}^{-}$are yet unknown. Here we will fill this gap. Moreover, the estimates obtained here (in Theorems 1, 2, 3) can be expanded to some non-linear functional differential equations (see Remark 1). Theorem 1 gives the best possible estimates of the norm of the Green operator for the periodic boundary value problem. In Theorem 2, we obtain unimprovable estimates of the solutions of (1)-(2) for non-negative $f$. In Theorem 3, unimprovable bounds of the difference between the maximum and the minimum of a solution are established.

We use the following notation: $\mathbb{R}$ is the space of real numbers, $\mathbf{C}$ is the space of continuous functions $x:[a, b] \rightarrow \mathbb{R}$ with the norm $\|x\|_{\mathbf{C}}=\max _{t \in[a, b]}|x(t)| ; \mathbf{L}$ is the space of integrable functions $z:[a, b] \rightarrow \mathbb{R}$ with the norm $\|z\|_{\mathbf{L}}=\int_{a}^{b}|z(t)| d t$; a linear bounded operator $T: \mathbf{C} \rightarrow \mathbf{L}$ is called positive if it maps non-negative functions from $\mathbf{C}$ into almost everywhere non-negative functions from $\mathbf{L}$.

Consider the periodic boundary value problem (1)-(2), where $f \in \mathbf{L}, T^{+}, T^{-}: \mathbf{C} \rightarrow \mathbf{L}$ are linear positive operators with norms $\mathcal{T}^{+} \equiv\left\|T^{+}\right\|_{\mathbf{C} \rightarrow \mathbf{L}}=\int_{a}^{b}\left(T^{+} \mathbf{1}\right)(t) d t, \mathcal{T}^{-} \equiv\left\|T^{-}\right\|_{\mathbf{C} \rightarrow \mathbf{L}}=$ $\int_{a}^{b}\left(T^{-} \mathbf{1}\right)(t) d t, \mathbf{1}$ is the unit function. An absolutely continuous function $x:[a, b] \rightarrow \mathbb{R}$ is called $a$ solution of the problem if it satisfies the periodic boundary condition (2) and equation (1) for almost all $t \in[a, b]$. We have to solve problem (1)-(2) if, for example, we search for periodic solutions of the equation with delay

$$
\dot{x}(t)=p(t) x(t-\tau(t))+f(t), \quad t \in \mathbb{R},
$$

where $p, f: \mathbb{R} \rightarrow \mathbb{R}$ are $(b-a)$-periodic locally integrable functions, $\tau: \mathbb{R} \rightarrow \mathbb{R}$ is a measurable $(b-a)$-periodic non-negative delay. Indeed, suppose that linear operators $T^{+}$and $T^{-}$are defined by the equalities

$$
\left(T^{+} x\right)(t)=\frac{p(t)+|p(t)|}{2} x(\tilde{\tau}(t)), \quad\left(T^{-} x\right)(t)=\frac{|p(t)|-p(t)}{2} x(\widetilde{\tau}(t)), \quad t \in[a, b],
$$

where $\widetilde{\tau}(t)=t-\tau(t)+k(t)(b-a)$ and the integer numbers $k(t)$ are such that $\widetilde{\tau}(t) \in[a, b]$ for almost all $t \in \mathbb{R}$. It is easy to show that problem (1)-(2) has a solution if and only if equation (5) has a periodic solution with the period $b-a$.

The conditions (3), (4) for the norms of the operators $T^{+}, T^{-}: \mathbf{C} \rightarrow \mathbf{L}$ are well known [15]. They guarantee the existence and uniqueness of solutions of problem (1)-(2). Note that these conditions are unimprovable in the following sense: if non-negative numbers $\mathcal{T}^{+}, \mathcal{T}^{-}$satisfy neither (3) nor (4), then problem (1)-(2) has no solution for some linear positive operators $T^{+}, T^{-}: \mathbf{C} \rightarrow \mathbf{L}$ with norms $\left\|T^{+}\right\|_{\mathbf{C} \rightarrow \mathbf{L}}=\mathcal{T}^{+},\left\|T^{-}\right\|_{\mathbf{C} \rightarrow \mathbf{L}}=\mathcal{T}^{-}$and for some $f \in \mathbf{L}$.

\section{The main results}

In what follows, we suppose that one of conditions (3), (4) is fulfilled. First, we formulate the results only for the simplest problem (1)-(2) with the null operator $T^{+}$:

$$
\begin{aligned}
& \dot{x}(t)=-\left(T^{-} x\right)(t)+f(t), \quad t \in[a, b], \\
& x(a)=x(b),
\end{aligned}
$$

where $T^{-}: \mathbf{C} \rightarrow \mathbf{L}$ is a linear positive operator with norm $\mathcal{T}^{-}, f \in \mathbf{L}$. The assertions of the following Theorems 1, 2, 3 for problem (6) are as follows. 
The solution $x$ of (6) satisfies the estimates

$$
\begin{aligned}
& \max _{t \in[a, b]}|x(t)| \leq \begin{cases}\frac{1+\mathcal{T}^{-}}{\mathcal{T}^{-}} \int_{a}^{b}|f(t)| d t & \text { if } 0<\mathcal{T}^{-} \leq 3 \\
\frac{4}{\mathcal{T}^{-}\left(4-\mathcal{T}^{-}\right)} \int_{a}^{b}|f(t)| d t & \text { if } 3<\mathcal{T}^{-}<4\end{cases} \\
& \max _{t \in[a, b]} x(t)-\min _{t \in[a, b]} x(t) \leq \begin{cases}\int_{a}^{b}|f(t)| d t & \text { if } 0<\mathcal{T}^{-} \leq 1, \\
\frac{1}{2 \sqrt{\mathcal{T}^{-}}-\mathcal{T}^{-}} \int_{a}^{b}|f(t)| d t & \text { if } 1<\mathcal{T}^{-}<4 .\end{cases}
\end{aligned}
$$

If a function $f$ is non-negative, the solution $x$ of (6) satisfies the estimates

$$
\begin{array}{ll}
\frac{1-\mathcal{T}^{-}}{\mathcal{T}^{-}} \int_{a}^{b} f(t) d t \leq x(t) \leq \frac{1+\mathcal{T}^{-}}{\mathcal{T}^{-}} \int_{a}^{b} f(t) d t & \text { if } 0<\mathcal{T}^{-} \leq 2 \\
-\frac{1}{4-\mathcal{T}^{-}} \int_{a}^{b} f(t) d t \leq x(t) \leq \frac{1+\mathcal{T}^{-}}{\mathcal{T}^{-}} \int_{a}^{b} f(t) d t & \text { if } 2<\mathcal{T}^{-} \leq 3 \\
-\frac{1}{4-\mathcal{T}^{-}} \int_{a}^{b} f(t) d t \leq x(t) \leq \frac{4}{\mathcal{T}^{-}\left(4-\mathcal{T}^{-}\right)} \int_{a}^{b} f(t) d t & \text { if } 3<\mathcal{T}^{-}<4
\end{array}
$$

All estimates (7), (8) and (9), which are proved in Theorems 1, 2, 3 in the general case, are best possible (see Remarks 3, 5, 6).

Remark 1 Consider also the non-linear periodic problem

$$
\begin{aligned}
& \dot{x}(t)=\left(F^{+} x\right)(t)-\left(F^{-} x\right)(t)+f(t), \quad t \in[a, b], \\
& x(a)=x(b),
\end{aligned}
$$

provided there exist non-negative functions $p^{+}, p^{-} \in \mathbf{L}$ with norms

$$
\left\|p^{+}\right\|_{\mathbf{L}}=\mathcal{T}^{+}, \quad\left\|p^{-}\right\|_{\mathbf{L}}=\mathcal{T}^{-}
$$

such that the operators $F^{+}, F^{-}: \mathbf{C} \rightarrow \mathbf{L}$ satisfy the inequalities

$$
\begin{array}{ll}
p^{+}(t) \min _{t \in[a, b]} x(t) \leq\left(F^{+} x\right)(t) \leq p^{+}(t) \max _{t \in[a, b]} x(t) & \text { for a.a. } t \in[a, b], \\
p^{-}(t) \min _{t \in[a, b]} x(t) \leq\left(F^{-} x\right)(t) \leq p^{-}(t) \max _{t \in[a, b]} x(t) & \text { for a.a. } t \in[a, b]
\end{array}
$$

for all $x \in \mathbf{C}$.

It follows from Lemma 3 and the proofs of Theorems 1, 2, 3 that all statements of these theorems are also valid for solutions of periodic problem (10)-(11) (if the solutions exist).

Theorem 1 If the norms $\mathcal{T}^{+}<\mathcal{T}^{-}$of the linear positive operators $T^{+}, T^{-}: \mathbf{C} \rightarrow \mathbf{L}$ satisfy the conditions

$$
3 \leq \mathcal{T}^{-}<2\left(1+\sqrt{1-\mathcal{T}^{+}}\right), \quad \mathcal{T}^{+}<3 / 4
$$

and $x$ is a solution of (1)-(2), then the inequality

$$
\max _{t \in[a, b]}|x(t)| \leq \frac{1}{\mathcal{T}^{-}\left(1-\mathcal{T}^{-} / 4\right)-\mathcal{T}^{+}} \int_{a}^{b}|f(t)| d t
$$

holds. 
If the norms $\mathcal{T}^{+}<\mathcal{T}^{-}$of the operators $T^{+}, T^{-}: \mathbf{C} \rightarrow \mathbf{L}$ satisfy

$$
\frac{\mathcal{T}^{+}}{1-\mathcal{T}^{+}}<\mathcal{T}^{-} \leq 3, \quad \mathcal{T}^{+}<3 / 4
$$

and $x$ is a solution of problem (1)-(2), then the inequality

$$
\max _{t \in[a, b]}|x(t)| \leq \frac{1+\mathcal{T}^{-}}{\mathcal{T}^{-}\left(1-\mathcal{T}^{+}\right)-\mathcal{T}^{+}} \int_{a}^{b}|f(t)| d t
$$

holds.

Remark 2 ([15]) If $\mathcal{T}^{-}>\mathcal{T}^{+} \geq 0$ and both of the conditions (15), (17) are not fulfilled, then there exist linear positive operators $T^{-}, T^{+}$with norms $\mathcal{T}^{-}, \mathcal{T}^{+}$and a function $f \in \mathbf{L}$ such that problem (1)-(2) has no solution.

Remark 3 From the proof of Theorem 1 it follows that estimates (16), (18) are best possible: if non-negative numbers $\mathcal{T}^{-}, \mathcal{T}^{+}$satisfy (15) (or (17)), then equality holds in condition (16) (or (18)) for a unique solution $x$ of problem (1)-(2) for some linear positive operators $T^{-}, T^{+}$with norms $\mathcal{T}^{-}, \mathcal{T}^{+}$and for some function $f \in \mathbf{L}, f \not \equiv 0$.

The estimates of solutions (1)-(2) for $\mathcal{T}^{-}<\mathcal{T}^{+}$can be obtained in the same way.

Theorem 1* If the norms $\mathcal{T}^{+}>\mathcal{T}^{-}$of the linear positive operators $T^{+}, T^{-}: \mathbf{C} \rightarrow \mathbf{L}$ satisfy the conditions

$$
3 \leq \mathcal{T}^{+}<2\left(1+\sqrt{1-\mathcal{T}^{-}}\right), \quad \mathcal{T}^{-}<3 / 4
$$

and $x$ is a solution of (1)-(2), then the inequality

$$
\max _{t \in[a, b]}|x(t)| \leq \frac{1}{\mathcal{T}^{+}\left(1-\mathcal{T}^{+} / 4\right)-\mathcal{T}^{-}} \int_{a}^{b}|f(t)| d t
$$

holds.

If the norms $\mathcal{T}^{+}>\mathcal{T}^{-}$of the operators $T^{+}, T^{-}: \mathbf{C} \rightarrow \mathbf{L}$ satisfy

$$
\frac{\mathcal{T}^{-}}{1-\mathcal{T}^{-}}<\mathcal{T}^{+} \leq 3, \quad \mathcal{T}^{-}<3 / 4
$$

and $x$ is a solution of problem (1)-(2), then the inequality

$$
\max _{t \in[a, b]}|x(t)| \leq \frac{1+\mathcal{T}^{+}}{\mathcal{T}^{+}\left(1-\mathcal{T}^{-}\right)-\mathcal{T}^{-}} \int_{a}^{b}|f(t)| d t
$$

holds.

Remark $2^{*}$ ([15]) If $\mathcal{T}^{-}>\mathcal{T}^{+} \geq 0$ and both of conditions (19), (21) are not fulfilled, then there exist linear positive operators $T^{-}$and $T^{+}$with norms $\mathcal{T}^{-}, \mathcal{T}^{+}$and a function $f \in \mathbf{L}$ such that problem (1)-(2) has no solution. 
Remark 3* From the proof of Theorem 1 it follows that estimates (20), (22) are best possible: if non-negative numbers $\mathcal{T}^{-}, \mathcal{T}^{+}$satisfy (19) (or (21)), then equality holds in condition (20) (or (22)) for a unique solution $x$ of problem (1)-(2) for some linear positive operators $T^{-}, T^{+}$with norms $\mathcal{T}^{-}, \mathcal{T}^{+}$and for some function $f \in \mathbf{L}, f \not \equiv 0$.

In the next statement we get the best possible lower bounds for solutions of problem (1)-(2) for non-negative $f$.

Theorem 2 Let $x$ be a solution of problem (1)-(2) for some non-negative $f$.

If the norms $\mathcal{T}^{+}, \mathcal{T}^{-}$of the operators $T^{+}, T^{-}: \mathbf{C} \rightarrow \mathbf{L}$ satisfy the conditions

$$
\max \left\{1+\sqrt{1-\mathcal{T}^{+}}, \frac{\mathcal{T}^{+}}{1-\mathcal{T}^{+}}\right\}<\mathcal{T}^{-}<2\left(1+\sqrt{1-\mathcal{T}^{+}}\right), \quad \mathcal{T}^{+}<3 / 4
$$

then

$$
\min _{t \in[a, b]} x(t) \geq-\frac{1}{2\left(1+\sqrt{1-\mathcal{T}^{+}}\right)-\mathcal{T}^{-}} \int_{a}^{b} f(t) d t
$$

if the norms $\mathcal{T}^{+}, \mathcal{T}^{-}$of the operators $T^{+}, T^{-}: \mathbf{C} \rightarrow \mathbf{L}$ satisfy the conditions

$$
\max \left\{1, \frac{\mathcal{T}^{+}}{1-\mathcal{T}^{+}}\right\}<\mathcal{T}^{-} \leq 1+\sqrt{1-\mathcal{T}^{+}},
$$

then

$$
\min _{t \in[a, b]} x(t) \geq-\frac{\mathcal{T}^{-}-1}{\mathcal{T}^{-}-\mathcal{T}^{+}} \int_{a}^{b} f(t) d t
$$

if the norms $\mathcal{T}^{+}, \mathcal{T}^{-}$of the operators $T^{+}, T^{-}: \mathbf{C} \rightarrow \mathbf{L}$ satisfy the conditions

$$
\frac{\mathcal{T}^{+}}{1-\mathcal{T}^{+}}<\mathcal{T}^{-} \leq 1, \quad \mathcal{T}^{+}<1 / 2
$$

then

$$
\min _{t \in[a, b]} x(t) \geq \frac{1-\mathcal{T}^{-}}{\mathcal{T}-\left(1+\mathcal{T}^{+}\right)-\mathcal{T}^{+}} \int_{a}^{b} f(t) d t
$$

Remark 4 ([15]) If $\mathcal{T}^{-}>\mathcal{T}^{+}$and all of conditions (23), (25), (27) are not fulfilled, then there exist linear positive operators $T^{-}$and $T^{+}$with norms $\mathcal{T}^{-}, \mathcal{T}^{+}$and a function $f \in \mathbf{L}$ such that problem (1)-(2) has no solution.

Remark 5 From the proof of Theorem 2 it follows that estimates (24), (26), (28) are best possible: if non-negative numbers $\mathcal{T}^{+}, \mathcal{T}^{-}$satisfy (23) ((25) or (27)), then equality holds in condition (24) ((26) or (28)) for a unique solution $x$ of problem (1)-(2) for some linear positive operators $T^{-}, T^{+}$with norms $\mathcal{T}^{-}, \mathcal{T}^{+}$and for some function $f \in \mathbf{L}, f \not \equiv 0$. 
Now we estimate the difference between the maximum and the minimum of solutions.

Theorem 3 Let the solvability conditions (4) be fulfilled and $x$ be a unique solution of (1)-(2). If

$$
\mathcal{T}^{-}>1, \quad \mathcal{T}^{+}<\mathcal{T}^{-}\left(\frac{\mathcal{T}^{-}-1}{\mathcal{T}^{-}+1}\right)^{2}
$$

then

$$
\max _{t \in[a, b]} x(t)-\min _{t \in[a, b]} x(t) \leq \frac{1}{2 \sqrt{\mathcal{T}^{-}-\mathcal{T}^{+}}-\mathcal{T}^{-}} \int_{a}^{b}|f(s)| d s
$$

otherwise

$$
\max _{t \in[a, b]} x(t)-\min _{t \in[a, b]} x(t) \leq \frac{\mathcal{T}^{-}}{\mathcal{T}^{-}-\mathcal{T}^{+}-\mathcal{T}^{-} \mathcal{T}^{+}} \int_{a}^{b}|f(s)| d s
$$

Remark 6 From the proof of Theorem 3 it follows that inequalities (29) and (30) are unimprovable. It means that for every number $\mathcal{T}^{+}, \mathcal{T}^{-}$satisfying the conditions of the theorem, equality holds in conditions (29) or (30) for the solution $x$ of problem (1)-(2) for some positive operators $T^{+}, T^{-}: \mathbf{C} \rightarrow \mathbf{L}$ with norms $\mathcal{T}^{-}, \mathcal{T}^{+}$, and for some non-negative function $f \in \mathbf{L}, f \not \equiv 0$.

Remark 7 Theorems 2, 3, as Theorem 1, can be easily reformulated for the case $\mathcal{T}^{+}>\mathcal{T}^{-}$ when the solvability condition (3) holds.

\section{Proofs}

We need three lemmas to prove the main theorems.

Lemma 1 Let $T^{+}, T^{-}: \mathbf{C} \rightarrow \mathbf{L}$ be linear positive operators, $p^{+}=T^{+} \mathbf{1}, p^{-}=T^{-} \mathbf{1}, y \in \mathbf{C}$. Then there exist points $t_{1}, t_{2} \in[a, b]$ and a function $p_{1} \in \mathbf{L}$ satisfying

$$
-p^{-}(t) \leq p_{1}(t) \leq p^{+}(t) \quad \text { for a.a. } t \in[a, b]
$$

such that the equality

$$
\left(T^{+} y\right)(t)-\left(T^{-} y\right)(t)=p_{1}(t) y\left(t_{1}\right)+\left(p^{+}(t)-p^{-}(t)-p_{1}(t)\right) y\left(t_{2}\right) \quad \text { for a.a. } t \in[a, b]
$$

holds.

Proof Let $y\left(t_{1}\right)=\max _{t \in[a, b]} y(t), y\left(t_{2}\right)=\min _{t \in[a, b]} y(t)$. Since $y \in \mathbf{C}$ and the linear operators $T^{+}, T^{-}: \mathbf{C} \rightarrow \mathbf{L}$ are positive, we have

$$
p^{+}(t) y\left(t_{2}\right)-p^{-}(t) y\left(t_{1}\right) \leq\left(T^{+} y\right)(t)-\left(T^{-} y\right)(t) \leq p^{+}(t) y\left(t_{1}\right)-p^{-}(t) y\left(t_{2}\right) \quad \text { for a.a. } t \in[a, b] .
$$

Therefore, for some function $p_{1} \in \mathbf{L}$ satisfying (31), equality (32) holds. 
Lemma 2 If $y \in \mathbf{C}$, functions $p^{+}, p^{-} \in \mathbf{L}$ are non-negative, and $p_{1} \in \mathbf{L}$ satisfies (31), then there exist linear positive operators $T^{+}, T^{-}: \mathbf{C} \rightarrow \mathbf{L}$ with the norms

$$
\left\|T^{+}\right\|_{\mathbf{C} \rightarrow \mathbf{L}}=\left\|p^{+}\right\|_{\mathbf{L}}, \quad\left\|T^{-}\right\|_{\mathbf{C} \rightarrow \mathbf{L}}=\left\|p^{-}\right\|_{\mathbf{L}}
$$

such that equality (32) holds.

Proof Let $p_{1}^{+}(t)=\left(\left|p_{1}(t)\right|+p_{1}(t)\right) / 2, p_{1}^{-}(t)=\left(\left|p_{1}(t)\right|-p_{1}(t)\right) / 2, t \in[a, b]$. Then the operators $T^{+}, T^{-}$defined by the equalities

$$
\begin{array}{ll}
\left(T^{+} x\right)(t)=p_{1}^{+}(t) x\left(t_{1}\right)+\left(p^{+}(t)-p_{1}^{+}(t)\right) x\left(t_{2}\right), & t \in[a, b], \\
\left(T^{-} x\right)(t)=p_{1}^{-}(t) x\left(t_{1}\right)+\left(p^{-}(t)-p_{1}^{-}(t)\right) x\left(t_{2}\right), & t \in[a, b],
\end{array}
$$

satisfy the conditions of the lemma.

Lemma 3 Let $F^{+}, F^{-}: \mathbf{C} \rightarrow \mathbf{L}$ satisfy (13)-(14), $y \in \mathbf{C}$. Then there exist a function $p_{1} \in \mathbf{L}$ satisfying (31) and points $t_{1}, t_{2} \in[a, b]$ such that the equality

$$
\left(F^{+} y\right)(t)-\left(F^{-} y\right)(t)=p_{1}(t) y\left(t_{1}\right)+\left(p^{+}(t)-p^{-}(t)-p_{1}(t)\right) y\left(t_{2}\right) \quad \text { for a.a. } t \in[a, b]
$$

holds.

Proof Let $y\left(t_{1}\right)=\max _{t \in[a, b]} y(t), y\left(t_{2}\right)=\min _{t \in[a, b]} y(t)$. Since $y \in \mathbf{C}$ and using (13), (14), we get

$$
p^{+}(t) y\left(t_{2}\right)-p^{-}(t) y\left(t_{1}\right) \leq\left(F^{+} y\right)(t)-\left(F^{-} y\right)(t) \leq p^{+}(t) y\left(t_{1}\right)-p^{-}(t) y\left(t_{2}\right) \quad \text { for a.a. } t \in[a, b] .
$$

Therefore, for some function $p_{1} \in \mathbf{L}$ satisfying (31), equality (34) holds.

Remark 8 It is obvious that one can choose the points $t_{1}$ and $t_{2}$ in Lemmas 1 and 3 in such a way that the solution $y$ takes its maximum and minimum at these points.

Proofs of Theorems 1, 2, 3 If $x$ is a solution of problem (1)-(2) ((10)-(11)), then by Lemma 1 (3) this solution satisfies the boundary value problem

$$
\begin{aligned}
& \dot{x}(t)=p_{1}(t) x\left(t_{1}\right)+\left(p^{+}(t)-p^{-}(t)-p_{1}(t)\right) x\left(t_{2}\right)+f(t), \quad t \in[a, b], \\
& x(a)=x(b),
\end{aligned}
$$

where $p_{1} \in \mathbf{L}$ and non-negative $p^{+}, p^{-} \in \mathbf{L}$ satisfy (31), (33). If condition (3) or (4) holds, then problem (35)-(36) has a unique solution, which can be easily found explicitly. Since we are only interested in the maximal and minimal values of the solutions, by Remark 8 , we have to obtain only representations for values $x\left(t_{1}\right)$ and $x\left(t_{2}\right)$.

Let $a \leq t_{1}<t_{2} \leq b, E \equiv\left[t_{1}, t_{2}\right], I \equiv\left[a, t_{1}\right] \cup\left[t_{2}, b\right]$,

$$
\begin{aligned}
\Delta \equiv & \int_{I} p_{1}(s) d s \int_{E}\left(p^{+}(s)-p^{-}(s)\right) d s-\int_{E} p_{1}(s) d s \int_{I}\left(p^{+}(s)-p^{-}(s)\right) d s \\
& -\int_{a}^{b}\left(p^{+}(s)-p^{-}(s)\right) d s .
\end{aligned}
$$


For $x\left(t_{1}\right), x\left(t_{2}\right)$ we have

$$
\begin{aligned}
x\left(t_{1}\right)= & \frac{1}{\triangle}\left(-\int_{I} f(s) d s \int_{E}\left(p^{+}(s)-p^{-}(s)-p_{1}(s)\right) d s\right. \\
& \left.+\int_{E} f(s) d s \int_{I}\left(p^{+}(s)-p^{-}(s)-p_{1}(s)\right) d s+\int_{a}^{b} f(s) d s\right) \\
x\left(t_{2}\right)= & \frac{1}{\triangle}\left(\int_{I} f(s) d s \int_{E} p_{1}(s) d s-\int_{E} f(s) d s \int_{I} p_{1}(s) d s+\int_{a}^{b} f(s) d s\right)
\end{aligned}
$$

and

$$
\begin{aligned}
x\left(t_{1}\right)-x\left(t_{2}\right)= & \frac{1}{\triangle}\left(-\int_{I} f(s) d s \int_{E}\left(p^{+}(s)-p^{-}(s)\right) d s\right. \\
& \left.+\int_{E} f(s) d s \int_{I}\left(p^{+}(s)-p^{-}(s)\right) d s\right) .
\end{aligned}
$$

Suppose here that $\mathcal{T}^{-}>\mathcal{T}^{+}$and condition (4) is fulfilled.

Define by $P$ the set of all solutions of problem (35)-(36) for all $a \leq t_{1}<t_{2} \leq b$, for all functions $p_{1} \in \mathbf{L}$ and non-negative $p^{+}, p^{-} \in \mathbf{L}$ such that conditions (12), (31) hold, and for all $f \in \mathbf{L}$ with $\|f\|_{\mathbf{L}}=1$.

Let $S$ be the subset of $P$ corresponding to non-negative functions $f$.

From Lemmas 1 and 2 , it follows that the set $P$ coincides with the set of all solutions of problem (1)-(2) for all linear positive operators $T^{-}, T^{+}: \mathbf{C} \rightarrow \mathbf{L}$ with norms $\left\|T^{+}\right\|_{\mathbf{C} \rightarrow \mathbf{L}}=\mathcal{T}^{+}$, $\left\|T^{-}\right\|_{\mathbf{C} \rightarrow \mathbf{L}}=\mathcal{T}^{-}$and for all $f \in \mathbf{L}$ with $\|f\|_{\mathbf{L}}=1$. The subset $S$ consists of all solutions of corresponding problems (1)-(2) with non-negative $f$.

Define the constants

$$
\begin{aligned}
& M_{1} \equiv \max _{x \in P, t \in[a, b]}|x(t)|, \quad M_{2} \equiv \max _{x \in P}\left(\max _{t \in[a, b]} x(t)-\min _{t \in[a, b]} x(t)\right), \\
& N_{1} \equiv \max _{x \in S, t \in[a, b]} x(t), \quad N_{2} \equiv \max _{x \in S}\left(\max _{t \in[a, b]} x(t)-\min _{t \in[a, b]} x(t)\right), \quad N_{3} \equiv \min _{x \in S, t \in[a, b]} x(t) .
\end{aligned}
$$

From representations (37), (38), (39), it easily follows that all the constants are defined correctly and

$$
M_{1}=\max \left\{\left|N_{1}\right|,\left|N_{3}\right|\right\}, \quad M_{2}=N_{2}
$$

Moreover, for every solution $x$ of (1)-(2), the following inequalities hold:

$$
\begin{aligned}
& |x(t)| \leq M_{1} \int_{a}^{b}|f(s)| d s, \quad t \in[a, b], \\
& \max _{t \in[a, b]} x(t)-\min _{t \in[a, b]} x(t) \leq N_{2} \int_{a}^{b}|f(s)| d s .
\end{aligned}
$$

If $f \in \mathbf{L}$ is non-negative, then

$$
N_{3} \int_{a}^{b} f(s) d s \leq x(t) \leq N_{1} \int_{a}^{b} f(s) d s, \quad t \in[a, b],
$$

where the constants $N_{1}, N_{2}, N_{3}, M_{1}$ are best possible. 
It remains to find $N_{1}, N_{2}, N_{3}$.

The numerator and denominator of fractions in (37), (38), (39) are linear with respect to variables $\int_{E} p_{1}(s) d s$ and $\int_{I} p_{1}(s) d s$. Therefore $x\left(t_{1}\right), x\left(t_{2}\right)$, and $x\left(t_{1}\right)-x\left(t_{2}\right)$ take their minimal and maximal values at the bounds of restriction (31) with respect to variables $p_{1}$ on each of the sets $E$ and $I$. Hence we have to consider only the following four different cases:

(i) $p_{1}(t)= \begin{cases}p^{+}(t) & \text { if } t \in E, \\ -p^{-}(t) & \text { if } t \in I\end{cases}$

(ii) $p_{1}(t)= \begin{cases}-p^{-}(t) & \text { if } t \in E \\ p^{+}(t) & \text { if } t \in I\end{cases}$

(iii) $p_{1}=p^{+}$,

(iv) $p_{1}=-p^{-}$.

In case (i) we have

$$
\begin{aligned}
& x\left(t_{1}\right)=\frac{1}{\triangle}\left(\int_{I} f(s) d s\left(\int_{E} p^{-}(s) d s+1\right)+\int_{E} f(s) d s\left(\int_{I} p^{+} d s+1\right)\right), \\
& x\left(t_{2}\right)=\frac{1}{\triangle}\left(\int_{I} f(s) d s\left(\int_{E} p^{+}(s) d s+1\right)+\int_{E} f(s) d s\left(\int_{I} p^{-} d s+1\right)\right), \\
& x\left(t_{1}\right)-x\left(t_{2}\right)=\frac{1}{\triangle}\left(\int_{I} f(s) d s\left(\int_{E}\left(p^{-}(s)-p^{+}(s)\right) d s\right)\right. \\
& \left.\quad-\int_{E} f(s) d s\left(\int_{I}\left(p^{-}(s)-p^{+}(s)\right) d s\right)\right), \\
& \triangle=\int_{I} p^{-}(s) d s \int_{E} p^{-}(s) d s-\int_{I} p^{+}(s) d s \int_{E} p^{+}(s) d s+\mathcal{T}^{-}-\mathcal{T}^{+} .
\end{aligned}
$$

In case (ii) we have

$$
\begin{aligned}
& x\left(t_{1}\right)=\frac{1}{\triangle}\left(\int_{I} f(s) d s\left(-\int_{E} p^{+}(s) d s+1\right)+\int_{E} f(s) d s\left(-\int_{I} p^{-}(s) d s+1\right)\right), \\
& x\left(t_{2}\right)=\frac{1}{\triangle}\left(\int_{I} f(s) d s\left(-\int_{E} p^{-}(s) d s+1\right)+\int_{E} f(s) d s\left(-\int_{I} p^{+}(s) d s+1\right)\right), \\
& x\left(t_{1}\right)-x\left(t_{2}\right)=\frac{1}{\triangle}\left(\int_{I} f(s) d s\left(\int_{E}\left(p^{-}(s)-p^{+}(s)\right) d s\right)\right. \\
& \left.-\int_{E} f(s) d s\left(\int_{I}\left(p^{-}(s)-p^{+}(s)\right) d s\right)\right), \\
& \triangle=\int_{I} p^{+}(s) d s \int_{E} p^{+}(s) d s-\int_{I} p^{-}(s) d s \int_{E} p^{-}(s) d s+\mathcal{T}^{-}-\mathcal{T}^{+} .
\end{aligned}
$$

In case (iii) we have

$$
\begin{aligned}
& x\left(t_{1}\right)=\frac{1}{\triangle}\left(\int_{I} f(s) d s\left(\int_{E} p^{-}(s) d s+1\right)+\int_{E} f(s) d s\left(-\int_{I} p^{-}(s) d s+1\right)\right), \\
& x\left(t_{2}\right)=\frac{1}{\triangle}\left(\int_{I} f(s) d s\left(\int_{E} p^{+}(s) d s+1\right)+\int_{E} f(s) d s\left(-\int_{I} p^{+}(s) d s+1\right)\right), \\
& x\left(t_{1}\right)-x\left(t_{2}\right)=\frac{1}{\triangle}\left(\int_{I} f(s) d s\left(\int_{E}\left(p^{-}(s)-p^{+}(s)\right) d s\right)\right. \\
& \left.-\int_{E} f(s) d s\left(\int_{I}\left(p^{-}(s)-p^{+}(s)\right) d s\right)\right), \\
& \triangle=-\int_{I} p^{+}(s) d s \int_{E} p^{-}(s) d s+\int_{I} p^{-}(s) d s \int_{E} p^{+}(s) d s+\mathcal{T}^{-}-\mathcal{T}^{+} .
\end{aligned}
$$


In case (iv) we have

$$
\begin{aligned}
& x\left(t_{1}\right)=\frac{1}{\triangle}\left(\int_{I} f(s) d s\left(-\int_{E} p^{+}(s) d s+1\right)+\int_{E} f(s) d s\left(\int_{I} p^{+}(s) d s+1\right)\right) \\
& x\left(t_{2}\right)=\frac{1}{\triangle}\left(\int_{I} f(s) d s\left(-\int_{E} p^{-}(s) d s+1\right)+\int_{E} f(s) d s\left(\int_{I} p^{-}(s) d s+1\right)\right) \\
& x\left(t_{1}\right)-x\left(t_{2}\right)=\frac{1}{\triangle}\left(\int_{I} f(s) d s\left(\int_{E}\left(p^{-}(s)-p^{+}(s)\right) d s\right)-\int_{E} f(s) d s\left(\int_{I}\left(p^{-}(s)-p^{+}(s)\right) d s\right)\right), \\
& \triangle=\int_{I} p^{+}(s) d s \int_{E} p^{-}(s) d s-\int_{I} p^{-}(s) d s \int_{E} p^{+}(s) d s+\mathcal{T}^{-}-\mathcal{T}^{+} .
\end{aligned}
$$

Let $S_{(\mathrm{i})}, S_{(\mathrm{ii})}, S_{(\mathrm{iii})}, S_{\text {(iv) }}$ be the subsets of $S$ for $p_{1}$ corresponding to cases (i), (ii), (iii), (iv).

We can easily calculate the minimal and maximal values in every case.

In case (iv) we have

$$
\begin{aligned}
& \max _{x \in S_{\text {(iv) }}}\left\{x\left(t_{1}\right), x\left(t_{2}\right)\right\}=\frac{\mathcal{T}^{-}+1}{\mathcal{T}^{-}-\mathcal{T}^{+}-\mathcal{T}^{-} \mathcal{T}^{+}}, \\
& \min _{x \in S_{(\text {iv) }}}\left\{x\left(t_{1}\right), x\left(t_{2}\right)\right\}= \begin{cases}\frac{1-\mathcal{T}^{-}}{\mathcal{T}^{-}-\mathcal{T}^{+}} & \text {if } \mathcal{T}^{-}>1, \\
\frac{1-\mathcal{T}^{-}}{\mathcal{T}^{-}-\mathcal{T}^{+}+\mathcal{T}^{-} \mathcal{T}^{+}} & \text {if } \mathcal{T}^{-} \leq 1,\end{cases} \\
& \min _{x \in S_{\text {(iv) }}}\left(x\left(t_{1}\right)-x\left(t_{2}\right)\right)=\frac{-\mathcal{T}^{-}}{\mathcal{T}^{-}-\mathcal{T}^{+}-\mathcal{T}^{-} \mathcal{T}^{+}}, \\
& \max _{x \in S_{(\text {(iv) }}}\left(x\left(t_{1}\right)-x\left(t_{2}\right)\right)= \begin{cases}\frac{\mathcal{T}^{-}}{\mathcal{T}^{-}-\mathcal{T}^{+}+\mathcal{T}^{-} \mathcal{T}^{+}} & \text {if } \mathcal{T}^{-}<1, \\
1 & \text { if } \mathcal{T}^{-} \geq 1 .\end{cases}
\end{aligned}
$$

In case (iii) we have

$$
\begin{aligned}
& \max _{x \in S_{(\text {iii) }}}\left\{x\left(t_{1}\right), x\left(t_{2}\right)\right\}=\frac{\mathcal{T}^{-}+1}{\mathcal{T}^{-}-\mathcal{T}^{+}-\mathcal{T}^{-} \mathcal{T}^{+}}, \\
& \min _{x \in S_{(\text {iii }}}\left\{x\left(t_{1}\right), x\left(t_{2}\right)\right\}= \begin{cases}\frac{1-\mathcal{T}^{-}}{\mathcal{T}^{-}-\mathcal{T}^{+}} & \text {if } \mathcal{T}^{-}>1, \\
\frac{1-\mathcal{T}^{-}}{\mathcal{T}^{-}-\mathcal{T}^{+}+\mathcal{T}^{-} \mathcal{T}^{+}} & \text {if } \mathcal{T}^{-} \leq 1,\end{cases} \\
& \max _{x \in S_{\text {(iii) }}}\left(x\left(t_{1}\right)-x\left(t_{2}\right)\right)=\frac{\mathcal{T}^{-}}{\mathcal{T}^{-}-\mathcal{T}^{+}-\mathcal{T}^{-} \mathcal{T}^{+}}, \\
& \min _{x \in S_{(\text {iii) }}}\left(x\left(t_{1}\right)-x\left(t_{2}\right)\right)= \begin{cases}\frac{-\mathcal{T}^{-}}{\mathcal{T}^{-}-\mathcal{T}^{+}+\mathcal{T}^{-} \mathcal{T}^{+}} & \text {if } \mathcal{T}^{-}<1, \\
-1 & \text { if } \mathcal{T}^{-} \geq 1 .\end{cases}
\end{aligned}
$$

Therefore, in cases (iii) and (iv) we have

$$
\begin{aligned}
& \max _{x \in S_{(\mathrm{iii})} \cup S_{(\mathrm{iv})}}\left\{x\left(t_{1}\right), x\left(t_{2}\right)\right\}=\frac{\mathcal{T}^{-}+1}{\mathcal{T}^{-}-\mathcal{T}^{+}-\mathcal{T}^{-} \mathcal{T}^{+}}, \\
& \min _{x \in S_{(\text {iii) }} \cup S_{(\text {iv) }}}\left\{x\left(t_{1}\right), x\left(t_{2}\right)\right\}= \begin{cases}\frac{1-\mathcal{T}^{-}}{\mathcal{T}^{-}-\mathcal{T}^{+}} & \text {if } \mathcal{T}^{-}>1, \\
\frac{1-\mathcal{T}^{-}}{\mathcal{T}^{-}-\mathcal{T}^{+}+\mathcal{T}^{-} \mathcal{T}^{+}} & \text {if } \mathcal{T}^{-} \leq 1,\end{cases} \\
& \max _{x \in S_{(\text {iii) }} \cup S_{(\text {iv) }}}\left|x\left(t_{1}\right)-x\left(t_{2}\right)\right|=\frac{\mathcal{T}^{-}}{\mathcal{T}^{-}-\mathcal{T}^{+}-\mathcal{T}^{-} \mathcal{T}^{+}} .
\end{aligned}
$$


In case (i) we have

$$
\begin{aligned}
& \max _{x \in S_{(\mathrm{i})}}\left\{x\left(t_{1}\right), x\left(t_{2}\right)\right\}=\frac{\mathcal{T}^{-}+1}{\mathcal{T}^{-}-\mathcal{T}^{+}-\left(\mathcal{T}^{+}\right)^{2} / 4}, \\
& \min _{x \in S_{(\mathrm{i})}}\left\{x\left(t_{1}\right), x\left(t_{2}\right)\right\}=\frac{1}{\mathcal{T}^{-}-\mathcal{T}^{+}+\left(\mathcal{T}^{-}\right)^{2} / 4}, \\
& \max _{x \in S_{(\mathrm{i})}}\left|x\left(t_{1}\right)-x\left(t_{2}\right)\right|=\max _{z \in\left[0, \mathcal{T}^{+}\right]}\left\{\frac{\mathcal{T}^{-}-z}{\mathcal{T}^{-}-\mathcal{T}^{+}-z\left(\mathcal{T}^{+}-z\right)}, \frac{z}{\mathcal{T}^{-}-\mathcal{T}^{+}-z\left(\mathcal{T}^{+}-z\right)}\right\} .
\end{aligned}
$$

In case (ii) we have

$$
\begin{aligned}
& \max _{x \in S_{(i i)}}\left\{x\left(t_{1}\right), x\left(t_{2}\right)\right\}=\frac{1}{\mathcal{T}^{-}-\mathcal{T}^{+}-\left(\mathcal{T}^{-}\right)^{2} / 4}, \\
& \min _{x \in S_{(i)}}\left\{x\left(t_{1}\right), x\left(t_{2}\right)\right\}= \begin{cases}\min \{K, G\} & \text { if } \mathcal{T}^{-} \leq 1, \\
\frac{1-\mathcal{T}^{-}}{\mathcal{T}^{-}-\mathcal{T}^{+}} & \text {if } 1<\mathcal{T}^{-} \leq 1+\sqrt{1-\mathcal{T}^{+}}, \\
-\frac{1}{2\left(1+\sqrt{1-\mathcal{T}^{+}}\right)-\mathcal{T}^{-}} & \text {if } 1+\sqrt{1-\mathcal{T}^{+}}<\mathcal{T}^{-},\end{cases}
\end{aligned}
$$

where $K=\min _{z \in\left[0, \mathcal{T}^{+}\right]} \frac{1-z}{\mathcal{T}^{-}-\mathcal{T}^{+}+z\left(\mathcal{T}^{+}-z\right)}, G=\min _{z \in\left[0, \mathcal{T}^{-}\right]} \frac{1-z}{\mathcal{T}^{-}-\mathcal{T}^{+}+\left(\mathcal{T}^{+}\right)^{2} / 4-z\left(\mathcal{T}^{-}-z\right)}$,

$$
\max _{x \in S_{(\mathrm{ii})}}\left|x\left(t_{1}\right)-x\left(t_{2}\right)\right|=\max _{z \in\left[0, \mathcal{T}^{-}\right]}\left\{\frac{z}{\mathcal{T}^{-}-\mathcal{T}^{+}-z\left(\mathcal{T}^{-}-z\right)}, \frac{\mathcal{T}^{+}-z}{\mathcal{T}^{-}-\mathcal{T}^{+}-z\left(\mathcal{T}^{-}-z\right)}\right\} .
$$

Considering extremal values in all cases (i), (ii), (iii) and (vi), by elementary calculation, we obtain

$$
\begin{aligned}
& N_{1}= \begin{cases}\frac{1}{\mathcal{T}^{-}-\mathcal{T}^{+}-\left(\mathcal{T}^{-}\right)^{2} / 4} & \text { if } \mathcal{T}^{-}>3, \\
\frac{\mathcal{T}^{-}+1}{\mathcal{T}^{-}-\mathcal{T}^{+}-\mathcal{T}^{-} \mathcal{T}^{+}} & \text {if } \mathcal{T}^{-} \leq 3,\end{cases} \\
& N_{3}= \begin{cases}\frac{1-\mathcal{T}^{-}}{\mathcal{T}^{-}-\mathcal{T}^{+}+\mathcal{T}^{-} \mathcal{T}^{+}} & \text {if } \mathcal{T}^{-} \leq 1, \\
\frac{1-\mathcal{T}^{-}}{\mathcal{T}^{-}-\mathcal{T}^{+}} & \text {if } 1<\mathcal{T}^{-} \leq 1+\sqrt{1-\mathcal{T}^{+}} \\
-\frac{1}{2\left(1+\sqrt{1-\mathcal{T}^{+}}\right)-\mathcal{T}^{-}} & \text {if } 1+\sqrt{1-\mathcal{T}^{+}}<\mathcal{T}^{-} .\end{cases}
\end{aligned}
$$

If $0 \leq \mathcal{T}^{+}<\mathcal{T}^{-}\left(1-\mathcal{T}^{-} / 4\right), 3 \leq \mathcal{T}^{-}$or $0 \leq \mathcal{T}^{+} \leq \frac{\mathcal{T}^{-}\left(\mathcal{T}^{-}-1\right)^{2}}{\left(\mathcal{T}^{-}+1\right)^{2}}, 1 \leq \mathcal{T}^{-} \leq 3$, then

$$
N_{2}=\frac{1}{2 \sqrt{\mathcal{T}^{-}-\mathcal{T}^{+}}-\mathcal{T}^{-}}
$$

If $0<\mathcal{T}^{+}<\frac{\mathcal{T}^{-}}{1+\mathcal{T}^{-}}, 0<\mathcal{T}^{-} \leq 1$ or $\frac{\mathcal{T}^{-}\left(\mathcal{T}^{-}-1\right)^{2}}{\left(\mathcal{T}^{-}+1\right)^{2}}<\mathcal{T}^{+}<\frac{\mathcal{T}^{-}}{1+\mathcal{T}^{-}}, 1<\mathcal{T}^{-} \leq 3$, then

$$
N_{2}=\frac{\mathcal{T}^{-}}{\mathcal{T}^{-}-\mathcal{T}^{+}-\mathcal{T}^{-\mathcal{T}^{+}}}
$$

This proves all Theorems 1, 2, 3 . 
Author's contributions

The author read and approved the final manuscript.

\section{Acknowledgements}

Research was supported by the Russian Foundation for Basic Research (14-01-0033814). The author would like to thank both reviewers for their careful reading of the manuscript and valuable remarks.

\section{Received: 29 January 2014 Accepted: 6 May 2014 Published: 15 May 2014}

\section{References}

1. Krawcewicz, W, Ma, S, Wu, J: Multiple slowly oscillating periodic solutions in coupled lossless transmission lines. Nonlinear Anal., Real World Appl. 5(2), 309-354 (2004)

2. Kang, S, Zhang, G: Existence of nontrivial periodic solutions for first order functional differential equations. Appl. Math. Lett. 18(1), 101-107 (2005)

3. Wu, J, Wang, Z: Periodic solutions of neutral functional differential systems with two parameters. Nonlinear Anal., Real World Appl. 9(3), 1012-1023 (2008)

4. Padhi, S, Srivastava, S: Multiple periodic solutions for a nonlinear first order functional differential equations with applications to population dynamics. Appl. Math. Comput. 203(1), 1-6 (2008)

5. Schwabik, S, Tvrdy, M, Vejvoda, O: Differential and Integral Equations. Boundary Value Problems and Adjoints. Czechoslovak Academy of Sciences, Dordrecht (1979)

6. Hale, JK, Verduyn Lunel, SM: Introduction to Functional Differential Equations. Springer, New York (1993)

7. Kolmanovskii, V, Myshkis, A: Introduction to the Theory and Applications of Functional Differential Equations. Kluwer Academic, Dordrecht (1999)

8. Kiguradze, I, Půža, B: Boundary Value Problems for Systems of Linear Functional Differential Equations. Masaryk University, Brno (2003)

9. Azbelev, NV, Maksimov, VP, Rakhmatullina, LF: Introduction to the Theory of Functional Differential Equations. Methods and Applications. Hindawi Publishing Corporation, New York (2007)

10. Skubachevskii, AL: Nonclassical boundary value problems. I. J. Math. Sci. (N.Y.) 155(2), 199-334 (2008)

11. Agarwal, RP, Berezansky, L, Braverman, E, Domoshnitsky, Al: Nonoscillation Theory of Functional Differential Equations with Applications. Springer, Berlin (2012)

12. Kiguradze, I, Půža, B: On periodic solutions to nonlinear functional-differential equations. Georgian Math. J. 6(1), 45-64 (1999)

13. Hakl, R, Lomtatidze, A, Šremr, J: On a periodic-type boundary value problem for first-order nonlinear functional differential equations. Nonlinear Anal., Theory Methods Appl. 51(3), 425-447 (2002)

14. Hakl, R, Lomtatidze, A, Půža, B: On periodic solutions of first order linear functional differential equations. Nonlinear Anal., Theory Methods Appl. 49(7), 929-945 (2002)

15. Hakl, R, Lomtatidze, A, Šremr, J: On a boundary-value problem of periodic type for first-order linear functional differential equations. Nonlinear Oscil. 5(3), 408-425 (2002)

16. Hakl, R, Lomtatidze, A, Šremr, J: Some Boundary Value Problems for First Order Scalar Functional Differential Equations. Masaryk University, Brno (2002)

17. Hakl, R, Lomtatidze, A, Šremr, J: On constant sign solutions of a periodic type boundary value problem for first order functional differential equations. Mem. Differ. Equ. Math. Phys. 26, 65-90 (2002)

18. Hakl, R, Lomtatidze, A, Šremr, J: Solvability and the unique solvability of a periodic type boundary value problem for first order scalar functional differential equations. Georgian Math. J. 9(3), 525-547 (2002)

19. Šremr, J, Šremr, P: On a two point boundary problem for first order linear differential equations with a deviating argument. Mem. Differ. Equ. Math. Phys. 29, 75-124 (2003)

20. Nieto, JJ, Rodríguez-López, R: Remarks on periodic boundary value problems for functional differential equations. J. Comput. Appl. Math. 158(2), 339-353 (2003)

21. Hakl, R, Lomtatidze, A, Šremr, J: Solvability of a periodic type boundary value problem for first order scalar functional differential equations. Arch. Math. 40(1), 89-109 (2004)

22. Nieto, JJ, Rodríguez-López, R: Monotone method for first-order functional differential equations. Comput. Math. Appl. 52(3-4), 471-484 (2006)

23. Bai, $D, X u, Y$ : Periodic solutions of first order functional differential equations with periodic deviations. Comput. Math. Appl. 53(9), 1361-1366 (2007) 